\title{
THE $\sigma$-CLASS GENERATED BY BALLS CONTAINS ALL BOREL SETS
}

\author{
VLADIMÍR OLEJČEK
}

(Communicated by Franklin D. Tall)

\begin{abstract}
The $\sigma$-class, i.e. the system of sets closed under complementation, countable disjoint unions and containing the empty set, generated by the system of open balls coincides with the $\sigma$-field of Borel sets in $\mathbb{R}^{3}$.
\end{abstract}

\section{INTRODUCTION}

Let $X$ be an arbitrary non-empty set. A class $\mathscr{L}$ of subsets of the set $X$, containing the empty set, is said to be $a \sigma$-class [3, p.672] (a concrete quantum logic [5, p.2], or a q- $\sigma$-algebra [4, p.121] ), if it is closed with respect to complementation and with respect to the union of any sequence of pairwise disjoint sets. If a $\sigma$-field of sets is defined in the usual way as a class of sets containing the empty set, closed with respect to complementation and with respect to unions of arbitrary sequences of sets, then, obviously, for an arbitrary class $\mathscr{C}$ of subsets of $X$, the $\sigma$-class $\mathscr{L}(\mathscr{C})$ generated by $\mathscr{C}$ is contained in the $\sigma$-field $\mathscr{A}(\mathscr{C})$ generated by $\mathscr{C}$. It is known [3, Corollary 1] that if $\mathscr{C}$ is closed with respect to intersection, i.e. if $A \in \mathscr{C}, B \in \mathscr{C}$ implies $A \cap B \in \mathscr{C}$, then $\mathscr{L}(\mathscr{C})=\mathscr{A}(\mathscr{C})$. Therefore, for instance, if $\mathscr{C}$ is the class of all (open) intervals on the real line, then $\mathscr{L}(\mathscr{C})=\mathscr{A}(\mathscr{C})$, where $\mathscr{A}(\mathscr{C})=\mathscr{B}$ is the class of Borel sets. For the same reason an analogous equality holds in the plane. In fact, if $\mathscr{C}$ is the class of all (open) rectangles, then $\mathscr{L}(\mathscr{C})$ coincides with the class $\mathscr{B}$ of all Borel sets in the plane. However, if $\mathscr{C}$ is taken to be the set of all discs (the fact they are open, closed or both is not essential), a question arises, whether $\mathscr{L}(\mathscr{C})$ contains all Borel sets, i.e. whether $\mathscr{L}(\mathscr{C})=\mathscr{B}$. More generally: Let $\mathscr{C}$ be the set of ( $n$-dimensional) open balls in the Euclidean space $\mathbb{R}^{n}$. Does $\mathscr{L}(\mathscr{C})$ equal $\mathscr{B}$ ? The question has been raised in even much more general form (for Banach algebras) by Preiss. However, it appeared to be non-trivial even in the above description, which was formulated independently by Neubrunn in 1977. The problem was positively answered in [4] for the two-dimensional case. This paper presents its solution in the three-dimensional space.

Received by the editors March 30, 1994.

1991 Mathematics Subject Classification. Primary 28A05, 04A03.

Key words and phrases. $\sigma$-class, concrete quantum logic, Borel sets, fractal, ball, net. 


\section{Preliminary lemmas and Definitions}

Lemma 1. Any $\sigma$-class $\mathscr{L}$ is closed with respect to intersections of decreasing sequences of sets.

Proof. See [2, Lemma 2.1].

Lemma 2. Let $\mathscr{C}$ be the class of all open balls in $\mathbb{R}^{3}$ and let $\mathscr{L}(\mathscr{C})$ be the $\sigma$-class generated by $\mathscr{C}$. Then

(1) every closed ball is contained in $\mathscr{L}(\mathscr{C})$,

(2) every one-point set is contained in $\mathscr{L}(\mathscr{C})$.

Proof. The properties of $\mathscr{L}(\mathscr{C})$ follow from Lemma 1.

We need to weaken the set inclusion and the set equality as follows.

Definition 1. We say that a set $A$ is a subset of a set $B \bmod \aleph_{0}$ (notation $A \subset B \bmod \aleph_{0}$ ), if there exists a countable set $C$ such that $A \subset B \cup C$. Two sets $A, B$ are called equal mod $\aleph_{0}\left(\right.$ notation $\left.A=B \bmod \aleph_{0}\right)$, if $A \subset B \bmod$ $\aleph_{0}$ and $B \subset A \bmod \aleph_{0}$.

Lemma 3. Let $\mathscr{C}$ be the class of all open balls in $\mathbb{R}^{3}$ and let $\mathscr{L}(\mathscr{C})$ be the $\sigma$-class generated by $\mathscr{C}$. Then any line segment I belongs to $\mathscr{L}(\mathscr{C})$.

Proof. For any positive integer $n$ we divide the set $I$ into $2^{n}$ equal parts $I_{n, i}$, $i=1,2, \ldots, 2^{n}$, and for each $i$ we denote by $L_{n, i}$ the open ball with the diameter $I_{n, i}$. The set $\bigcup_{i=1}^{2^{n}} L_{n, i}$ belongs to $\mathscr{L}(\mathscr{C})$, thus, according to Lemma 1, $\bigcap_{n=1}^{\infty} \bigcup_{i=1}^{2^{n}} L_{n, i}$ belongs to $\mathscr{L}(\mathscr{C})$. Since $I=\bigcap_{n=1}^{\infty} \bigcup_{i=1}^{2^{n}} L_{n, i} \bmod \aleph_{0}$, according to Lemma 2, (2) I also belongs to $\mathscr{L}(\mathscr{C})$.

Definition 2. Two sets $D$ and $E$ from a system $\mathscr{C}$ are said to be compatible with respect to $\mathscr{C}$, if $D \cap E \in \mathscr{C}$ or $D \cap E=\varnothing$.

Definition 3. A class of sets is called a net, if any two sets in it are either disjoint or one is contained in the other $([1, p .14])$.

Orthogonality in concrete quantum logics usually corresponds to disjointness. However, in this paper it is used exclusively in the geometrical sense.

Definition 4. Two balls (or discs) are called orthogonal, if the square of the distance of their centres is equal to the sum of the squares of their radii.

Definition 5. Two nets $\mathscr{Z}, \mathscr{Y}$ of balls (or discs) are said to be orthogonal nets, if any two balls (discs) $A \in \mathscr{X}, B \in \mathscr{Y}$ are compatible or orthogonal.

\section{A fRActal STRUCTURE IN $\mathbb{R}^{2}$}

In this section we define a fractal structure in $\mathbb{R}^{2}$, which enables us to prove (in Section 5) that any square in $\mathbb{R}^{3}$ belongs to $\mathscr{L}(\mathscr{C})$. The system $\mathscr{C}$ is taken to be the system of all open discs. Considering Cartesian coordinates let us define

$$
\operatorname{inv}(x, y)=\left(\frac{2 x}{x^{2}+y^{2}}, \frac{2 y}{x^{2}+y^{2}}\right)
$$


a transformation in the plane, which is commonly known as the "circle inversion'. It is determined by a circle, in this particular example by the circle with the centre $(0,0)$ and the radius $\sqrt{ } 2$. It is also known that the transformation inv

(1) is a conformal transformation, i.e. it preserves the size of angles,

(2) is an involution, i.e. $\operatorname{inv}(\operatorname{inv}(x, y))=(x, y)$,

(3) transforms a circle to a circle or to a line depending on whether it contains the origin or not.

Any open disc $D$ in $\mathbb{R}^{2}$ is represented by a triple $\left(x_{1}, x_{2}, r\right)$, where $x_{1}, x_{2}$ are the coordinates of the centre and $r$ is the radius. As usual, $\operatorname{inv}(D)$ denotes the image of the disc $D$ by the map inv. Let $\mathscr{X}$ be any set of discs in $\mathbb{R}^{2}$. Denote

$$
\begin{aligned}
\operatorname{ext}(\mathscr{X})= & \left\{E=\left(y_{1}, y_{2}, r\right): 0 \leq y_{i}=2 p_{i}+s_{i} x_{i},\right. \\
& D=\left(x_{1}, x_{2}, r\right) \in \mathscr{X}, p_{i} \in\{0,1,2, \ldots\}, \\
& \left.\operatorname{not} p_{1}=p_{2}=0, s_{i} \in\{-1,1\}, i=1,2\right\},
\end{aligned}
$$

and

$$
\begin{aligned}
\operatorname{inv}(\mathscr{X})= & \left\{E=\left(y_{1}, y_{2}, r\right): 0 \leq y_{i} \leq 1, i=1,2,\right. \\
& E=\operatorname{inv}(D), D \in \mathscr{X}\} .
\end{aligned}
$$

Further, put $\mathscr{B}_{1}=\left\{B_{1}\right\}$, where $B_{1}=(0,1,1), \mathscr{G}_{1}=\left\{G_{1}\right\}$, where $G_{1}=$ $(1,0,1)$, and for any integer $k>1$

$$
\mathscr{Y}_{k+1}=\operatorname{inv}\left(\operatorname{ext}\left(\mathscr{X}_{k}\right)\right) \text {, }
$$

where $\mathscr{Y}=\mathscr{G}$ or $\mathscr{B}$, if $\mathscr{X}=\mathscr{B}$ or $\mathscr{G}$ respectively. Finally, denote $\mathscr{B}_{\infty}=$ $\bigcup_{k=1}^{\infty} \mathscr{B}_{k}$ and $\mathscr{G}_{\infty}=\bigcup_{k=1}^{\infty} \mathscr{G}_{k}$. Figure 1 (on the next page) shows the discs from $\mathscr{B}_{k}$ (black circles) and from $\mathscr{G}_{k}$ (grey circles) for $k=1, \ldots, 6$ with radii not smaller than 0.01 .

Using the properties of the transformations inv and ext and applying the standard routine of induction, the following lemma can be proved

Lemma 4. $\mathscr{B}_{\infty}$ and $\mathscr{G}_{\infty}$ are orthogonal nets.

\section{A COVER OF A SQUARE}

Now an essential step follows to prove that any closed square in $\mathbb{R}^{3}$ belongs to $\mathscr{L}(\mathscr{C})$.

Lemma 5. Let $B_{n}=\bigcup \mathscr{B}_{n}, G_{n}=\bigcup \mathscr{G}_{n}$ and

$$
G_{n, i}=\bigcup\left\{D \in \mathscr{G}_{n}: D \nsubseteq B_{i}\right\}
$$

for any positive integers $i, n$ and let

$$
Q=\{(x, y): 0 \leq x \leq 1,0 \leq y \leq 1\}
$$

Then

and

$$
Q=\bigcap_{k=1}^{\infty} \bigcup_{n=k}^{\infty} B_{n} \cup \bigcup_{k=1}^{\infty} \bigcap_{n=k}^{\infty} \bigcap_{i=k}^{n} G_{n, i} \bmod \aleph_{0}
$$

$$
\bigcap_{k=1}^{\infty} \bigcup_{n=k}^{\infty} B_{n} \cap \bigcup_{k=1}^{\infty} \bigcap_{n=k}^{\infty} \bigcap_{i=k}^{n} G_{n, i}=\varnothing
$$




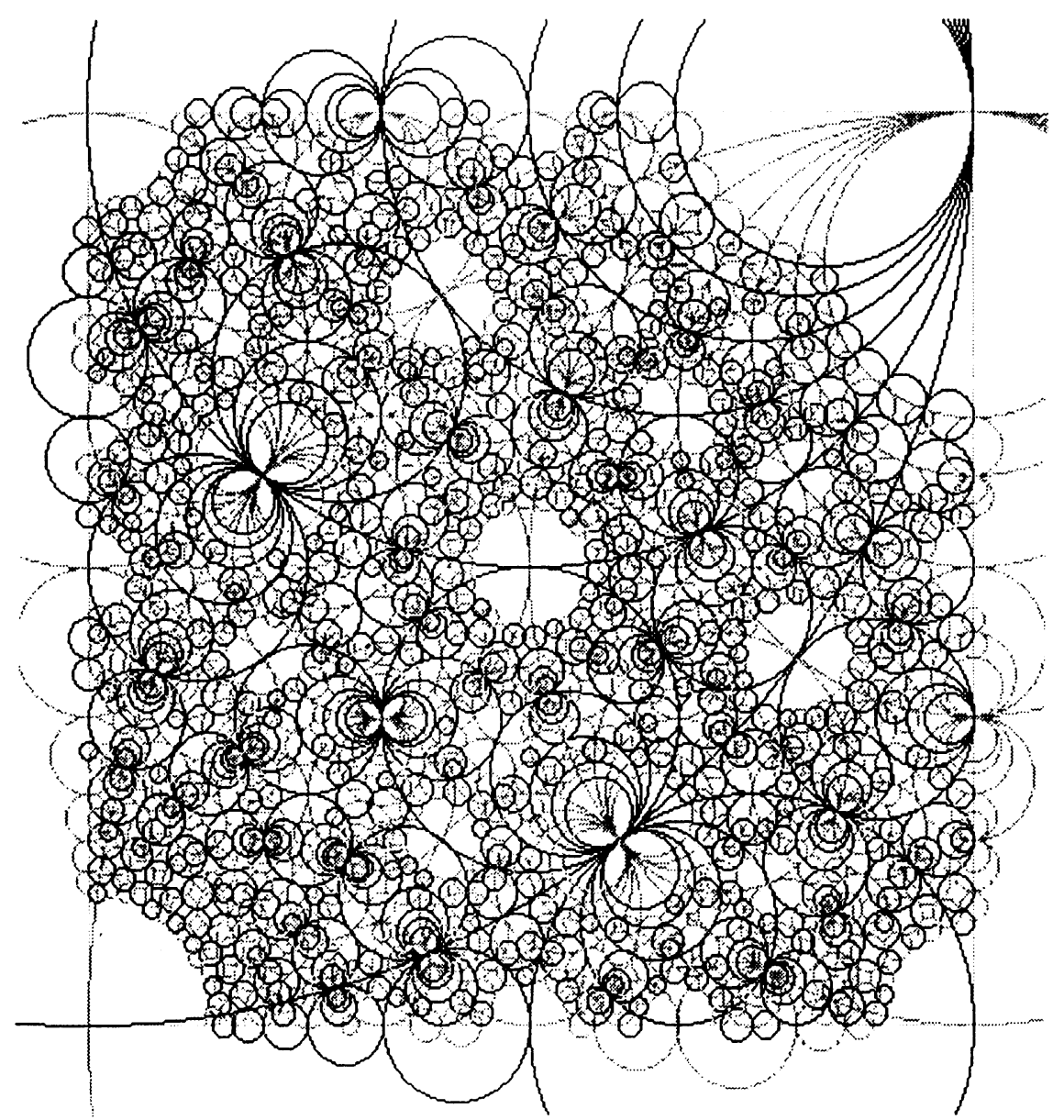

FIGURE 1

Proof. Taking an arbitrary positive integer $n$ we have

$$
Q \subset B_{n} \cup G_{n} \bmod \aleph_{0}
$$

and since $G_{n, k} \cap G_{n, k+1} \cap \cdots \cap G_{n, n}$ contains all discs from $\mathscr{G}_{n}$ which are not completely covered by $B_{k} \cup B_{k+1} \cup \cdots \cup B_{n}$, we have also

$$
Q \subset B_{k} \cup B_{k+1} \cup \cdots \cup B_{n} \cup\left[G_{n, k} \cap G_{n, k+1} \cap \cdots \cap G_{n, n}\right] \bmod \aleph_{0}
$$

for any integers $k \leq n$.

Figure 2 describes the idea of the covering construction for $k=1$ and $n=1,2,3$ (first column), and for $k=2$ and $n=2,3,4$ (second column). The black (grey) circles represent discs forming $B_{i}\left(G_{n, i}\right)$ with radii exceeding 0.01 . 

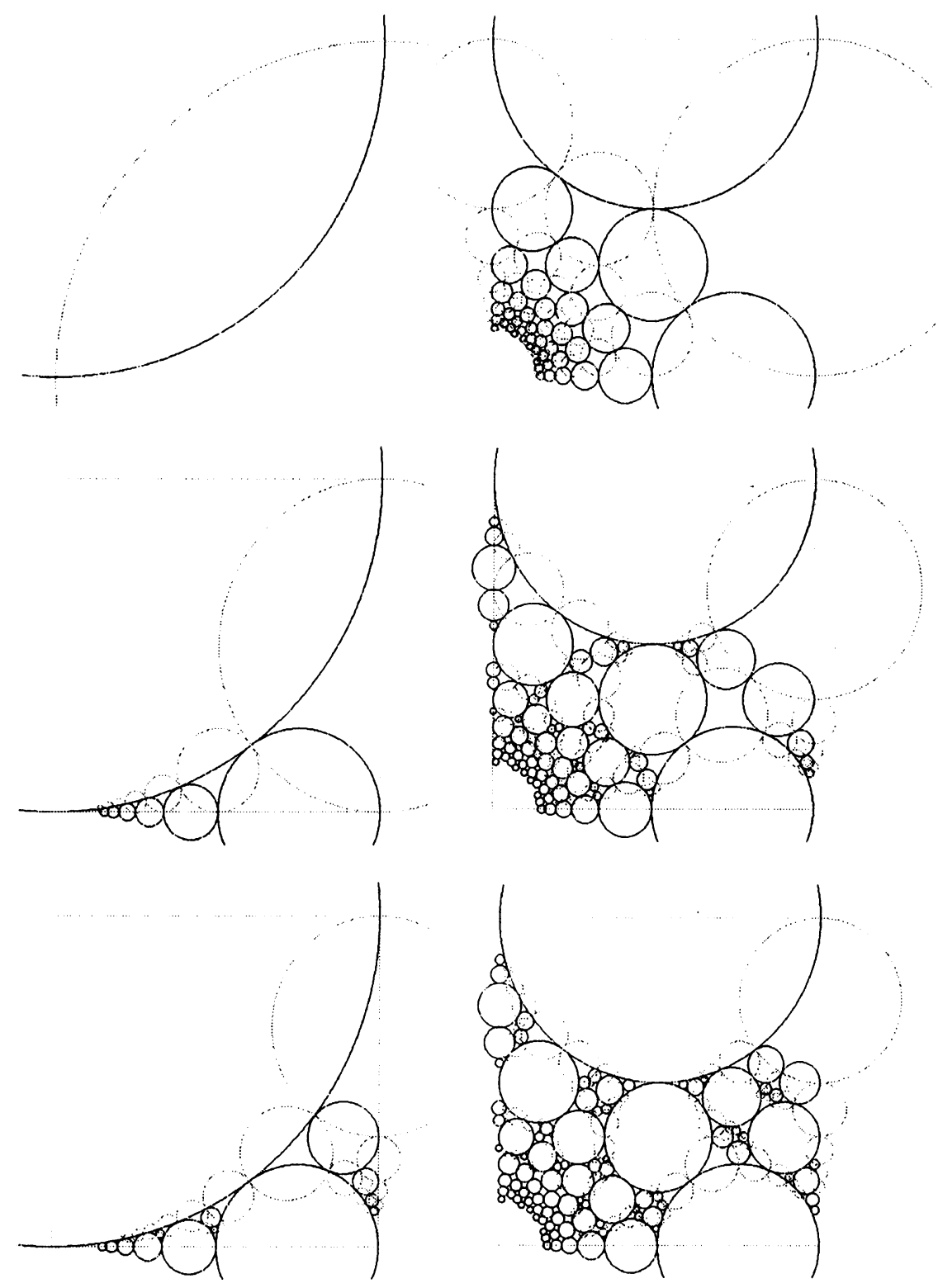

FIGURE 2

From the last inclusion it follows

$$
Q \subset \bigcup_{n=k}^{\infty} B_{n} \cup \bigcap_{i=k}^{n} G_{n, i} \bmod \aleph_{0}
$$

for any $k \leq n$. Since the sequence $\bigcap_{i=k}^{n} G_{n, i}$ is decreasing with respect to $n$, we have

$$
Q \subset \bigcup_{n=k}^{\infty} B_{n} \cup \bigcap_{n=k}^{\infty} \bigcap_{i=k}^{n} G_{n, i} \bmod \aleph_{0}
$$


for any $k$. Hence

$$
Q \subset \bigcup_{n=k}^{\infty} B_{n} \cup \bigcup_{k=1}^{\infty} \bigcap_{n=k}^{\infty} \bigcap_{i=k}^{n} G_{n, i} \bmod \aleph_{0}
$$

for any $k$ and since the sequence $\bigcup_{n=k}^{\infty} B_{n}$ is decreasing with respect to $k$, we have finally

$$
Q \subset \bigcap_{k=1}^{\infty} \bigcup_{n=k}^{\infty} B_{n} \cup \bigcup_{k=1}^{\infty} \bigcap_{n=k}^{\infty} \bigcap_{i=k}^{n} G_{n, i} \bmod \aleph_{0}
$$

To prove the opposite inclusion and the disjointness, we use the following property of the systems $\mathscr{B}_{n}, \mathscr{G}_{n}$, which is not difficult to verify: If $\mathrm{r}_{n}$ denotes the maximum of the radii of all discs contained in $\mathscr{B}_{n} \cup \mathscr{G}_{n}$, then $\lim _{n} \mathrm{r}_{n}=0$ (in fact $\mathrm{r}_{n}=\frac{1}{n}$ ).

If a point $p$ does not belong to $Q$, then at least one of its coordinates is outside the interval $[0,1]$ and therefore there exists a positive integer $n(p)$ such that $p \notin B_{n} \cup G_{n}$ for all $n \geq n(p)$. Hence

$$
p \notin \bigcap_{k=1}^{\infty} \bigcup_{n=k}^{\infty} B_{n} \cup \bigcup_{k=1}^{\infty} \bigcap_{n=k}^{\infty} \bigcap_{i=k}^{n} G_{n, i}
$$

Finally, let $p \in \bigcap_{k=1}^{\infty} \bigcup_{n=k}^{\infty} B_{n}$. Then, for any positive integer $k$, an integer $n(k)$ can be found such that $p \in B_{n(k)}$. Let $d_{k}$ denote the distance of $p$ from the boundary of $B_{n(k)}$. Since $B_{n(k)}$ is a disjoint union of open discs, $d_{k}$ is strictly positive. Then, for any positive integer $k$, there exists such an integer $m(k)$ that the radii of all discs from $\mathscr{G}_{m(k)}$ are smaller than $d_{k}$ and simultaneously $m(k) \geq n(k)$. Considering the orthogonality of overlapping discs from $\mathscr{B}_{n(k)}$ and $\mathscr{G}_{n(k)}$, we have

$$
p \notin G_{m(k), n(k)} \Rightarrow p \notin \bigcap_{i=k}^{m(k)} G_{m(k), i} \Rightarrow p \notin \bigcap_{n=k}^{\infty} \bigcap_{i=k}^{n} G_{n, i}
$$

for an arbitrary $k$, and consequently

$$
p \notin \bigcup_{k=1}^{\infty} \bigcap_{n=k}^{\infty} \bigcap_{i=k}^{n} G_{n, i}
$$

Note that for every $k, \bigcup_{n=k}^{\infty} B_{n}$ is a sort of 'osculatory packing' [1, p.125] and $\bigcap_{n=k}^{\infty} \bigcap_{i=k}^{n} G_{n, i}$ is the residual set ' $\bmod \aleph_{0}$ '.

\section{CONSEQUENCES IN $\mathbb{R}^{2}$ AND IN $\mathbb{R}^{3}$}

Lemma 6. If $\mathscr{C}$ is the system of all open discs in $\mathbb{R}^{2}$, and $\mathscr{L}(\mathscr{C})$ is the $\sigma$-class generated by $\mathscr{C}$, then $\mathscr{L}(\mathscr{C})$ contains any closed square in $\mathbb{R}^{2}$.

Proof. We can introduce the coordinate system in $\mathbb{R}^{2}$ such that $(0,0),(1,0)$, $(1,1)$ and $(0,1)$ are the vertices of the square. According to Lemma 4

$$
\bigcap_{k=1}^{\infty} \bigcup_{n=k}^{\infty} B_{n} \in \mathscr{L}(\mathscr{C}) \quad \text { and } \quad \bigcup_{k=1}^{\infty} \bigcap_{n=k}^{\infty} \bigcap_{i=k}^{n} G_{n, i} \in \mathscr{L}(\mathscr{C}) \text {. }
$$


Since, according to Lemma 2, one-point sets belong to $\mathscr{L}(\mathscr{C})$, the unit square $Q$ belongs to $\mathscr{L}(\mathscr{C})$ as well.

Let us return to $\mathbb{R}^{3}$. Let $\mathscr{C}$ be again the set of all open balls in $\mathbb{R}^{3}$ and let $\mathscr{L}(\mathscr{C})$ be the respective generated $\sigma$-class. Our next goal is to prove that any square in $\mathbb{R}^{3}$ belongs to $\mathscr{L}(\mathscr{C})$. Let a coordinate system be introduced in $\mathbb{R}^{3}$ such that a closed square $Q$ has vertices $(0,0,0),(1,0,0),(1,1,0)$ and $(0,1,0)$. We can construct a fractal structure in $\mathbb{R}^{3}$ copying the procedure in Section 3 , taking open balls instead of open discs such that the third coordinates of their centres equal 0 . There is no change in the definitions of the transformations inv and ext apart from the third 0-coordinate added. Analogous assertions to Lemmas 4 and 5 can be proved and they imply the following consequence.

Lemma 7. The $\sigma$-class $\mathscr{L}(\mathscr{C})$ generated by the system $\mathscr{C}$ of all open balls in $\mathbb{R}^{3}$ contains any closed square.

\section{A FRACTAL STRUCTURE IN $\mathbb{R}^{3}$}

The key problem of our final target is to prove that a closed cube in $\mathbb{R}^{3}$ belongs to $\mathscr{L}(\mathscr{C})$. To some extent it is analogous to the procedures in Sections 3 and 4.

We define a transformation inv in $\mathbb{R}^{3}$ as follows:

$$
\operatorname{inv}(x, y)=\left(\frac{2 x}{x^{2}+y^{2}+z^{2}}, \frac{2 y}{x^{2}+y^{2}+z^{2}}, \frac{2 z}{x^{2}+y^{2}+z^{2}}\right) .
$$

It is a 'spherical inversion' determined by a sphere with the radius $\sqrt{ } 2$ and with the centre in the origin and it has similar properties to those of the circle inversion, i.e. it preserves angles, it is an involution and it transforms spheres to spheres with some exceptions.

Any ball $S$ in $\mathbb{R}^{3}$ can be represented by a quadruple $\left(x_{1}, x_{2}, x_{3}, r\right)$, where $x_{1}, x_{2}, x_{3}$ are coordinates of the centre and $r$ is the radius. As above, $\operatorname{inv}(S)$ denotes the image of the set $S$ of the set $S \in \mathbb{R}^{3}$ in the transformation. Let $\mathscr{X}$ be any set of balls in $\mathbb{R}^{3}$. We define

$$
\begin{aligned}
\operatorname{ext}(\mathscr{X})= & \left\{T=\left(y_{1}, y_{2}, y_{3}, r\right): 0 \leq y_{i}=2 p_{i}+s_{i} x_{i}\right. \\
& S=\left(x_{1}, x_{2}, x_{3}, r\right) \in \mathscr{X}, p_{i} \in\{0,1,2, \ldots\}, \\
& \text { not } \left.p_{1}=p_{2}=p_{3}=0, s_{i} \in\{-1,1\}, i=1,2,3\right\},
\end{aligned}
$$

and

$$
\begin{aligned}
\operatorname{inv}(\mathscr{X})= & \left\{T=\left(y_{1}, y_{2}, y_{3}, r\right): 0 \leq y_{i} \leq 1, i=1,2,3,\right. \\
& T=\operatorname{inv}(S), S \in \mathscr{X}\} .
\end{aligned}
$$

Further, put $\mathscr{P}_{1}=\left\{P_{1}\right\}$, where $P_{1}=(1,1,1,1), \mathscr{W}_{1}=\left\{W_{1}\right\}$, where $W_{1}=$ $(0,0,1,1), \mathscr{B}_{1}=\left\{B_{1}\right\}$, where $B_{1}=(0,1,0,1), \mathscr{G}_{1}=\left\{G_{1}\right\}$, where $G_{1}=$ $(1,0,0,1)$. For any integer $k>1$ we define four systems $\mathscr{P}_{k}, \mathscr{W}_{k}, \mathscr{B}_{k}, \mathscr{G}_{k}$, by induction

$$
\mathscr{Y}_{k+1}=\operatorname{inv}\left(\operatorname{ext}\left(\mathscr{X}_{k}\right)\right)
$$

where, if $\mathscr{X}=\mathscr{P}$ then $\mathscr{Y}=\mathscr{P}$ and further it depends on whether $k$ is odd or even: in case $k$ is even

$$
\mathscr{X}=\mathscr{B} \Rightarrow \mathscr{Y}=\mathscr{W}, \quad \mathscr{X}=\mathscr{G} \Rightarrow \mathscr{Y}=\mathscr{B}, \quad \mathscr{X}=\mathscr{W} \Rightarrow \mathscr{Y}=\mathscr{G},
$$


in case $k$ is odd

$$
\mathscr{Z}=\mathscr{B} \Rightarrow \mathscr{Y}=\mathscr{G}, \quad \mathscr{Z}=\mathscr{G} \Rightarrow \mathscr{Y}=\mathscr{W}, \quad \mathscr{X}=\mathscr{W} \Rightarrow \mathscr{Y}=\mathscr{B} .
$$

Finally, denote $\mathscr{X}_{\infty}=\bigcup_{k=1}^{\infty} \mathscr{X}_{k}$ for all $\mathscr{Z} \in\{\mathscr{P}, \mathscr{W}, \mathscr{B}, \mathscr{G}\}$. Using the properties of the transformations inv and ext the following lemma (analogous to Lemma 4) can be proved by induction.

Lemma 8. The systems of balls $\mathscr{P}_{\infty}, \mathscr{W}_{\infty}, \mathscr{B}_{\infty}$ and $\mathscr{G}_{\infty}$ are pairwise orthogonal nets.

\section{A COVER OF A CUBE}

A 'covering' lemma, partially analogous to Lemma 5, follows.

Lemma 9. Let

$$
Q=\{(x, y, z): 0 \leq x \leq 2,0 \leq y \leq 2,0 \leq z \leq 2\}
$$

and for any triple $(u, v, w) \in\{0,1\}^{3}$ define a transformation

$$
\operatorname{turn}_{u, v, w}(x, y, z)=\left(2 u+(-1)^{u} x, 2 v+(-1)^{v} y, 2 w+(-1)^{w} z\right) .
$$

Denote $X_{n}=\bigcup \mathscr{X}_{n}$, for all $X \in\{P, W, B, G\}, \mathscr{X} \in\{\mathscr{P}, \mathscr{W}, \mathscr{B}, \mathscr{E}\}$ and

$$
\begin{aligned}
B_{n, i} & =\bigcup\left\{S \in \mathscr{B}_{n}: S \nsubseteq W_{i}\right\}, \\
G_{n, i, j} & =\bigcup\left\{S \in \mathscr{G}_{n}: S \nsubseteq W_{i}, S \nsubseteq B_{j}\right\}
\end{aligned}
$$

for any positive integers $i, j, n$. Further denote

$$
\begin{aligned}
P_{\infty} & =\bigcup_{m=1}^{\infty} P_{m}, \\
W_{\infty} & =\bigcap_{p=1}^{\infty} \bigcup_{i=p}^{n} W_{i}, \\
B_{\infty} & =\bigcap_{p=1}^{\infty} \bigcup_{q=p}^{\infty} \bigcap_{k=q}^{\infty} \bigcup_{n=k}^{\infty} \bigcap_{i=p}^{n} B_{n, i}, \\
G_{\infty} & =\bigcup_{p=1}^{\infty} \bigcap_{q=p}^{\infty} \bigcup_{k=q}^{\infty} \bigcap_{n=k}^{n} \bigcap_{j=k}^{n} \bigcap_{i=p}^{n} G_{n, i, j} .
\end{aligned}
$$

Then

$$
Q=\bigcup\left\{\operatorname{turn}_{u, v, w}\left(P_{\infty} \cup W_{\infty} \cup B_{\infty} \cup G_{\infty}\right):(u, v, w) \in\{0,1\}^{3}\right\} \bmod \aleph_{0}
$$

and the sets $P_{\infty}, W_{\infty}, B_{\infty}$, and $G_{\infty}$ are pairwise disjoint.

Proof. First we denote

$$
Q_{1}=\{(x, y, z): 0 \leq x \leq 1,0 \leq y \leq 1,0 \leq z \leq 1\},
$$

and prove that

$$
Q_{1} \subset P_{\infty} \cup W_{\infty} \cup B_{\infty} \cup G_{\infty} \bmod \aleph_{0} .
$$

For an arbitrary integer $p$ we have

$$
Q_{1} \subset \bigcup_{m=1}^{p} P_{m} \cup W_{p} \cup B_{p, p} \cup G_{p, p, p} \bmod \aleph_{0}
$$


whence, for arbitrary $p, n, p \leq n$,

$$
Q_{1} \subset \bigcup_{m=1}^{n} P_{m} \cup \bigcup_{i=p}^{n} W_{i} \cup \bigcap_{i=p}^{n} B_{n, i} \cup \bigcap_{i=p}^{n} G_{n, i, n} \bmod \aleph_{0}
$$

Obviously, $n$ in the unions can be replaced by $\infty$, therefore

$$
Q_{1} \subset \bigcup_{m=1}^{\infty} P_{m} \cup \bigcup_{i=p}^{\infty} W_{i} \cup \bigcap_{i=p}^{n} B_{n, i} \cup \bigcap_{i=p}^{n} G_{n, i, n} \bmod \aleph_{0}
$$

Using a consideration similar to that one in the proof of Lemma 5 , taking $\bigcap_{i=p}^{n} B_{n, i}$ on the position of $B_{n}$ and $\bigcap_{i=p}^{n} G_{n, i, n}$ on the position of $G_{n}$ in (*), we obtain

$$
Q_{1} \subset \bigcup_{m=1}^{\infty} P_{m} \cup \bigcup_{i=p}^{\infty} W_{i} \cup \bigcap_{k=q}^{\infty} \bigcup_{n=k}^{\infty} \bigcap_{i=p}^{n} B_{n, i} \cup \bigcup_{k=q}^{\infty} \bigcap_{n=k}^{\infty} \bigcap_{j=k}^{n} \bigcap_{i=p}^{n} G_{n, i, j} \bmod \aleph_{0},
$$

for arbitrary $p, q, p \leq q$. In the third term we can take its union through all $q$ and since the fourth term decreases with respect to $q$, it can be replaced by the intersection. For arbitrary $p$ we have

$$
Q_{1} \subset \bigcup_{m=1}^{\infty} P_{m} \cup \bigcup_{i=p}^{\infty} W_{i} \cup \bigcup_{q=p}^{\infty} \bigcap_{k=q}^{\infty} \bigcup_{n=k}^{\infty} \bigcap_{i=p}^{n} B_{n, i} \cup \bigcap_{q=p}^{\infty} \bigcup_{k=q}^{\infty} \bigcap_{n=k}^{\infty} \bigcap_{j=k}^{n} \bigcap_{i=p}^{n} G_{n, i, j} \bmod \aleph_{0}
$$

Finally, since the second and the third term are decreasing and the last term is increasing with respect to $p$, we obtain the desired inclusion

$$
\begin{array}{r}
Q_{1} \subset \bigcup_{m=1}^{\infty} P_{m} \cup \bigcap_{p=1}^{\infty} \bigcup_{i=p}^{\infty} W_{i} \cup \bigcap_{p=1}^{\infty} \bigcup_{q=p}^{\infty} \bigcap_{k=q}^{\infty} \bigcup_{n=k}^{\infty} \bigcap_{i=p}^{n} B_{n, i} \\
\cup \bigcup_{p=1}^{\infty} \bigcap_{q=p}^{\infty} \bigcup_{k=q}^{\infty} \bigcap_{n=k}^{\infty} \bigcap_{j=k}^{n} \bigcap_{i=p}^{n} G_{n, i, j} \bmod \aleph_{0} .
\end{array}
$$

It is not difficult to verify that

$$
Q \subset \bigcup\left\{\operatorname{turn}_{u, v, w}\left(Q_{1}\right):(u, v, w) \in\{0,1\}^{3}\right\}
$$

whence

$$
Q \subset \bigcup\left\{\operatorname{turn}_{u, v, w}\left(P_{\infty} \cup W_{\infty} \cup B_{\infty} \cup G_{\infty}\right):(u, v, w) \in\{0,1\}^{3}\right\} .
$$

To prove the opposite inclusion note that the radii of balls belonging to $\mathscr{P}_{n} \cup \mathscr{W}_{n} \cup \mathscr{B}_{n} \cup \mathscr{G}_{n}$ tend to 0 as $n$ approaches $\infty$ also in the three-dimensional construction. It follows that the inclusion

$$
Q_{1} \supset W_{\infty} \cup B_{\infty} \cup G_{\infty}
$$

can be proved in the same way as the related part of Lemma 5 . It is also clear that any ball from $\mathscr{P}_{\infty}$ is contained in $Q$ and $\operatorname{since} \operatorname{turn}_{u, v, w}(Q)=Q$ for any $(u, v, w) \in\{0,1\}^{3}$, we have

$$
Q \supset \bigcup\left\{\operatorname{turn}_{u, v, w}\left(P_{\infty} \cup W_{\infty} \cup B_{\infty} \cup G_{\infty}\right):(u, v, w) \in\{0,1\}^{3}\right\} .
$$

The idea of the proof of the pairwise disjointness of the sets $P_{\infty}, W_{\infty}, B_{\infty}$ and $G_{\infty}$ is also the same as in the proof of Lemma 5. The construction of 
$\mathscr{P}_{\infty}, \mathscr{W}_{\infty}, \mathscr{B}_{\infty}$ and $\mathscr{G}_{\infty}$ directly shows that an arbitrary ball in $\mathscr{P}_{\infty}$ contains no ball from $\mathscr{W}_{\infty} \cup \mathscr{B}_{\infty} \cup \mathscr{G}_{\infty}$ as a subset. It follows that any point $x \in P_{\infty}$ may appear only in a finite number of balls from $\mathscr{W}_{\infty} \cup \mathscr{B}_{\infty} \cup \mathscr{G}_{\infty}$. Consequently, $P_{\infty}$ has no common points with any of the sets $W_{\infty}, B_{\infty}$ and $G_{\infty}$.

Assume that $x \in W_{\infty}=\bigcap_{p=1}^{\infty} \bigcup_{i=p}^{n} W_{i}$. Then for any integer $p$ there exists an integer $i(p)$ such that $x \in W_{i(p)}$. Denote by $d_{p}$ the distance of $x$ from the boundary of $W_{i(p)}$. Since the radii of balls in $\mathscr{B}_{n} \cup \mathscr{G}_{n}$ decrease to 0 as $n$ approaches $\infty$, there exists an index $n(p)$ such that for any $n, n \geq n(p)$, the radii of all balls in $\mathscr{B}_{n} \cup \mathscr{G}_{n}$ are smaller than $d_{p}$. Since $\mathscr{W}, \mathscr{B}$ and $\mathscr{W}, \mathscr{G}$ are orthogonal pairs of nets, $x \notin B_{n, i(p)}$ and $x \notin G_{n, i(p), j}$ for any $j$. Without any restriction we can assume that $n(p) \geq n$, whence

$$
x \notin \bigcap_{p=1}^{\infty} \bigcup_{q=p}^{\infty} \bigcap_{k=q}^{\infty} \bigcup_{n=k}^{\infty} \bigcap_{i=p}^{n} B_{n, i} \quad \text { and } \quad x \notin \bigcup_{p=1}^{\infty} \bigcap_{q=p}^{\infty} \bigcup_{k=q}^{\infty} \bigcap_{n=k}^{\infty} \bigcap_{j=k}^{n} \bigcap_{i=p}^{n} G_{n, i, j} .
$$

The disjointness of $B_{\infty}$ and $G_{\infty}$ can be proved similarly as in the related part of the proof of Lemma 5.

Corollary. The $\sigma$-class $\mathscr{L}(\mathscr{C})$ generated by the system $\mathscr{C}$ of all open balls contains every closed cube.

Proof. We prove that the cube $Q$ from Lemma 9 belongs to $\mathscr{L}(\mathscr{C})$. For that purpose it is enough to show that for an arbitrary pair of triples $\left(u_{1}, v_{1}, w_{1}\right)$, $\left(u_{2}, v_{2}, w_{2}\right) \in\{0,1\}^{3}$ the set

$$
\operatorname{turn}_{u_{1}, v_{1}, w_{1}}\left(P_{\infty} \cup W_{\infty} \cup B_{\infty} \cup G_{\infty}\right) \cap \operatorname{turn}_{u_{2}, v_{2}, w_{2}}\left(P_{\infty} \cup W_{\infty} \cup B_{\infty} \cup G_{\infty}\right)
$$

belongs to $\mathscr{L}(\mathscr{C})$. Moreover, we can restrict consideration to two pairs $(0,0,0),(0,0,1)$ and $(0,0,0),(0,1,1)$ due to symmetry. (For the pair $(0,0,0),(1,1,1)$ the above intersection equals $\left.P_{1} \in \mathscr{L}(\mathscr{C}).\right)$ For any $X \in$ $\{P, W, B, G\}$ denote by $X_{\infty}^{1}$ the set constructed the same way as $X_{\infty}$ restricting the set of used balls to those with the third coordinate of the center equal to 1 , and $X_{\infty}^{2}$ the set constructed using only the balls with the second and third coordinate of the center equal to 1 . Then

$$
\begin{gathered}
\operatorname{turn}_{0,0,0}\left(P_{\infty} \cup W_{\infty} \cup B_{\infty} \cup G_{\infty}\right) \cap \operatorname{turn}_{0,0,1}\left(P_{\infty} \cup W_{\infty} \cup B_{\infty} \cup G_{\infty}\right) \\
=P_{\infty}^{1} \cup W_{\infty}^{1} \cup B_{\infty}^{1} \cup G_{\infty}^{1}
\end{gathered}
$$

and

$$
\begin{gathered}
\operatorname{turn}_{0,0,0}\left(P_{\infty} \cup W_{\infty} \cup B_{\infty} \cup G_{\infty}\right) \cap \operatorname{turn}_{0,1,1}\left(P_{\infty} \cup W_{\infty} \cup B_{\infty} \cup G_{\infty}\right) \\
=P_{\infty}^{2} \cup W_{\infty}^{2} \cup B_{\infty}^{2} \cup G_{\infty}^{2} .
\end{gathered}
$$

Since for $i=1,2$ the sets $P_{\infty}^{i}, W_{\infty}^{i}, B_{\infty}^{i}, G_{\infty}^{i}$ are pairwise disjoint and each of them belongs to $\mathscr{L}(\mathscr{C})$, the intersection belongs to $\mathscr{L}(\mathscr{C})$ as well.

\section{THE MAIN ResUlt}

Theorem. The $\sigma$-class $\mathscr{L}(\mathscr{C})$ generated by the system $\mathscr{C}$ of all open balls coincides with the $\sigma$-field $\mathscr{B}$ of Borel sets in $\mathbb{R}^{3}$.

Proof. Obviously $\mathscr{L}(\mathscr{C}) \subset \mathscr{B}$. To prove the opposite inclusion it suffices to show that every closed block (three-dimensional interval) belongs to $\mathscr{L}(\mathscr{C})$. 
From Lemma 8 it follows that $P_{\infty}, W_{\infty}, B_{\infty}$, and $G_{\infty}$ belong to $\mathscr{L}(\mathscr{C})$. According to Corollary of Lemma 9 , every closed cube belongs to $\mathscr{L}(\mathscr{C})$. Since, according to Lemma $7, \mathscr{L}(\mathscr{C})$ contains every closed square and, according to Lemma 3, it contains every line segment, any closed block with rational sizes can be formed as a union of closed cubes within $\mathscr{L}(\mathscr{C})$. Therefore, any closed block with rational sizes belongs to $\mathscr{L}(\mathscr{C})$. Finally, forming decreasing sequences of closed blocks with rational sizes and applying Lemma 1 we obtain that every closed block is contained in $\mathscr{L}(\mathscr{C})$.

\section{REFERENCES}

1. K. J. Falconer, The geometry of fractal sets, Cambridge Univ. Press, Cambridge and New York, 1985.

2. S. P. Gudder, Quantum probability spaces, Proc. Amer. Math. Soc. 21 (1969), 296-302.

3. T. Neubrunn, A note on quantum probability spaces, Proc. Amer. Math. Soc. 25 (1970), 672-675.

4. V. Olejček, Generation of a $q-\sigma$-algebra in the plane, Proc. Conf. Topology and Measure V, Wissenshaftliche Beiträge der Ernst-Moritz-Arndt-Universität Greifswald, 1988, pp. 121-125.

5. P. Pták and S. Pulmannová, Orthomodular structures as quantum logics, Kluwer Acad. Publ., Dordrecht, 1991.

Department of Mathematics, Faculty of Electrical ENGineering and Information Technology, Slovak Technical University, Ilkovicova 3, SK-81219 BratislaVa, Slovakia

E-mail address: olejcek@kmat.elf.stuba.sk 